\title{
An interesting case report of ruptured pyogenic liver abscess with 40 weeks gestation in labour
}

\author{
Shraddha A. Mevada*, Archana A. Bhosale, Sayali Wankhedkar, Rucha Choudhari
}

Department of Obstetrics and Gynecology, Lokmanya Tilak Municipal Medical College and General Hospital, Sion, Mumbai, Maharashtra, India

Received: 15 March 2020

Accepted: 04 April 2020

*Correspondence:

Dr. Shraddha A. Mevada,

E-mail: drmevadashraddha@gmail.com

Copyright: (c) the author(s), publisher and licensee Medip Academy. This is an open-access article distributed under the terms of the Creative Commons Attribution Non-Commercial License, which permits unrestricted non-commercial use, distribution, and reproduction in any medium, provided the original work is properly cited.

\begin{abstract}
Pyogenic liver abscess during pregnancy is an extremely rare condition. Although rare, in situations of sepsis or septic shock in pregnancy, as well as the common sources of infection, a possibility of a liver abscess should be considered. We present a case report of 32-year-old primigravida 37.3 weeks by date, 37 weeks by scan with breech presentation with premature rupture of membranes and pain in abdomen since 12 hours with breathlessness, fever and diarrhoea since 2 days came in emergency to study hospital. General condition of the patient on arrival was pulse-140 beats per minute, blood pressure was $90 / 60 \mathrm{mmHg}$, respiratory rate was $40 / \mathrm{min}$, on per abdominal examination, breech presentation with fetal heart rate of 150 beats per minute on doppler was noted. Per vaginal examination revealed cervical os $5 \mathrm{~cm}$ dilated, $40 \%$ effacement, breech presentation, absent membranes. With urgent report of complete hemogram and acid blood gas analysis, metabolic acidosis was noted which was corrected and patient was taken for emergency lower segment caesarean section. Intra-operative, $250 \mathrm{ml}$ greenish pus flakes fluid was noted inside the abdominal cavity. Fluid was drained and sent for culture sensitivity with maximum aseptic precautions, uterus was opened, baby was delivered followed by uterus closure. Ruptured liver abscess $6 \times 4 \times 2 \mathrm{~cm}$ in $2^{\text {nd }}$ and $3^{\text {rd }}$ segment of liver was noted, abdominal wash with antibiotics and NS was given, drain was kept. Appropriate antibiotics were started and was discharged on day 14 after suture removal.
\end{abstract}

Keywords: Breathlessness, Breech, Primigravidae, Ruptured liver abscess, Sepsis

\section{INTRODUCTION}

Pyogenic liver abscess (PLA) complicating pregnancy is extremely rare. The most common microorganisms reported with this complication are Escherichia coli and Bacteroides spp. and polymicrobial infections. ${ }^{1}$ Adequate management of this unusual clinical situation requires early diagnosis and treatment.

\section{CASE REPORT}

A 32-year-old primigravida 37.3 weeks by date, 37 weeks by scan with breech presentation with premature rupture of membranes and pain in abdomen since 12 hours with breathlessness, fever and diarrhea since 2 days came in emergency unit to study hospital. Patient had 1 episode of fever at home, 3-4 episodes of loose stools since 2 days, and breathlessness NYHA grade 2 since 2 days.

General condition of the patient on arrival was pulse-140 beats per minute, blood pressure was 110/80 $\mathrm{mmHg}$, respiratory rate was $40 / \mathrm{min}$, oxygen saturation was $87 \%$. Cardiovascular system-S1S2 +, respiratory system, -air entry bilaterally equal, normal.

On per abdominal examination breech presentation with fetal heart rate of 150 beats per minute on Doppler was noted, with good contraction of 3-4 /10 minutes/35 sec. 
Per vaginal examination revealed cervical os $5 \mathrm{~cm}$ dilated, $40 \%$ effacement, footling breech presentation, absent membranes.

She already had ultrasonography report of single live intrauterine gestation with breech presentation with estimated fetal weight $3.76 \mathrm{~kg}$ and amniotic fluid index of 2 .

With urgent report of complete hemogram which stated leukocytosis with WBC count of $23,800 / \mathrm{mm}^{3}$ was and acid blood gas analysis, metabolic acidosis was noted which was corrected.

\section{Other investigations were as follows}

CBC-hemoglobin: 11.2, total leucocyte count: 23,800, platelet count: $3,48,000$, LFT-total bilirubin: 0.3 , SGOT:21, SGPT:17, Na+/K+- 141.6/5.01, RFT-blood urea nitrogen: 8.8 , creatinine: 0.9 , C-reactive protein: 87.07.

Sinus tachycardia on ECG was noted. Portable X-ray chest showed normal finding.

Patient was taken for emergency lower segment caesarean section in view of primigravida with footling breech presentation in active labour.

\section{Outcomes}

On opening the peritoneal cavity, $250 \mathrm{ml}$ greenish pus flakes fluid was noted. Fluid was drained and $10 \mathrm{ml}$ of fluid was sent for culture and sensitivity. With maximum aseptic precautions, i.e., with 3 mop technique, uterus was opened by Kerrs incision, baby was delivered, followed by uterus closure. Further incision was extended vertically to localise the source of septic foci. Ruptured liver abscess $6 \times 4 \times 2 \mathrm{~cm}$ in $2^{\text {nd }}$ and $3^{\text {rd }}$ segment of liver was noted which was drained. With the help of surgeons, abdominal wash with metronidazole and normal saline was given followed by intraperitoneal drain insertion. Appropriate higher spectrum antibiotics were started.

Patient required intensive care unit admission and ventilatory support for 24 hours. Patient improved gradually after a course of antibiotics and serial monitoring, with gradual decrease in leucocyte counts.

Pus culture sensitivity report suggested Klebsiella species sensitive to Piperacillin-Tazobactam. No growth was noted in blood culture sensitivity report. Patient was discharged on day 14 after suture removal with healthy mother and baby

\section{DISCUSSION}

Pyogenic liver abscess is a serious, life threatening condition that is difficult to diagnose. Clinical suspicion is important because of its high mortality rate. ${ }^{1}$ The most common symptoms are fever and right upper quadrant abdominal pain, the clinical presentation in many cases is nonspecific and is difficult to diagnose. The most common microorganisms reported with this clinical entity are Escherichia coli and Bacteroides spp. ${ }^{1}$ Usually, a mucosal defect within the digestive tract is blamed for bacteria invasion into the portal system followed by hematogenous spread to the liver. ${ }^{2}$ Until now, few cases are noted associated with pregnancy.

Just like the clinical symptoms, the laboratory tests are also non-specific for diagnosis of PLA. The most frequent findings are increased ALP, leukocytosis, and increased fibrinogen which are not specific during pregnancy. Elevated ALT and infection-induced thrombocytopenia are also reported. ${ }^{3}$ The sensitivity of ultrasound for the diagnosis of PLA is reported to be $85.8 \% .^{4}$ The clinical incidence of PLA varies from region to region but has been reported to be 11 cases per million persons per year. ${ }^{5}$

PLA during pregnancy is an extremely rare condition which represents a diagnostic and therapeutic challenge. As the clinical and laboratory findings are usually nonspecific, a misdiagnosis is often possible, but an early diagnosis and therapy are vital because of its high perinatal mortality rate in untreated cases. Another problem with PLA onset in pregnancy is the possible progression of the disease to severe sepsis or septic shock which is associated with increased rates of preterm delivery, fetal infection, multiple organ dysfunction syndrome, and death.

Sepsis is the situation of systemic inflammatory response syndrome (SIRS) because of an infection. SIRS is defined as the presence of two or more of the following: temperature greater than $38^{\circ} \mathrm{C}$ or less than $36^{\circ} \mathrm{C}$, pulse greater than 90 beats/min, respiratory rate greater than 20 breaths/min, partial carbon monoxide pressure less than $32 \mathrm{mmHg}$, and white blood cell count greater than $12,000 / \mathrm{mm}^{3}$ or less than $4,000 / \mathrm{mm}^{3}{ }^{6}$ Sometimes, SIRS and sepsis may progress to multiple organ dysfunction and septic shock, which is a more serious and mortal complication.

As the sepsis and septic shock during pregnancy can be fatal, an immediate empiric initiation of a large spectrum antibiotic treatment is vital. But even with the appropriate antibiotic selection and adequate fluid resuscitation, the prognosis is poor, unless the target of the infection is found. Especially in cases of abscess formation in certain tissues or organs, the treatment can be useless if the source of the infection is not drained or excised.

Differential diagnosis of sepsis is important. It tends to occur from specific sources, such as respiratory infections, that are the most common causes of sepsis, genitourinary, and abdominal sources of infection with primary bacteremia and unknown sources being the next most common causes. ${ }^{7}$ In pregnancy, premature rupture 
of membranes and chorioamnionitis also should be considered.

In this case, the patient suffered from gastroenteritis before her admission to study hospital. At first, all study attempts of finding a septic focus failed. But when we examined the right upper quadrant, we could identify a pyogenic abscess in the liver. This case was successfully treated with a proper diagnosis and prompt treatment. After her discharge, her routine visits revealed normal results and she gave birth to a healthy full-term baby.

Although rare, in situations of sepsis or septic shock in pregnancy, as well as the common sources of infection, a possibility of a liver abscess should also be kept in mind and ultrasonographic evaluation of this region should be considered.

\section{Funding: No funding sources}

Conflict of interest: None declared

Ethical approval: The study was approved by the Institutional Ethics Committee

\section{REFERENCES}

1. Pérez JA, González JJ, Baldonedo RF, Sanz L, Carreño G, Junco A, et al. Clinical course, treatment, and multivariate analysis of risk factors for pyogenic liver abscess. Am J Surg. 2001;181(2):177-86.
2. Cohen JL, Martin FM, Schoetz DJ. Liver abscess. The need for complete gastrointestinal evaluation. Arch Surg. 1989;124(5):561-4.

3. Zhu X, Wang S, Jacob R, Zhang F, Ji G. A 10-year retrospective analysis of clinical profiles, laboratory characteristics and management of pyogenic liver abscesses in a Chinese hospital. Gut Liver. 2011;5(2):221-7.

4. Lin AC, Yeh DY, Hsu YH, Wu CC, Chang H, Jang $\mathrm{TN}$, et al. Diagnosis of pyogenic liver abscess by abdominal ultrasonography in the emergency department. Emerg Med J. 2009;26(4):273-5.

5. Khanna N, Inkster T. Meticillin-resistant Staphylococcus aureus hepatic abscess treated with tigecycline. J Clin Pathol. 2008;61(8):967-8.

6. Barton JR, Sibai BM. Severe sepsis and septic shock in pregnancy. Obstet Gynecol. 2012;120(3):689-706.

7. Martin GS. Sepsis, severe sepsis and septic shock: changes in incidence, pathogens and outcomes. Exp Rev Anti-Infect Ther. 2012;10(6):701-6.

Cite this article as: Mevada SA, Bhosale AA, Wankhedkar S, Choudhari R. An interesting case report of ruptured pyogenic liver abscess with 40 weeks gestation in labour. Int J Reprod Contracept Obstet Gynecol 2020;9:2205-7. 